\title{
Understanding the Experiences of Heavy Smokers after Exercise
}

\author{
Mary Hassandra1, Athanasios Kolovelonis ${ }^{2}$, Stiliani Ani Chroni' ${ }^{3}$, Alkistis Olympiou ${ }^{4}$, \\ Marios Goudas ${ }^{2}$, Yiannis Theodorakis ${ }^{2}$ \\ ${ }^{1}$ Department of Sports, University of Jyväskylä, Jyväskylä, Finland \\ ${ }^{2}$ Department of Physical Education \& Sport Science, University of Thessaly, Trikala, Greece \\ ${ }^{3}$ Department of Sports \& Physical Education, Hedmark University College, Elverum, Norway \\ ${ }^{4}$ School of Sport and Exercise Science, College of Social Science, University of Lincoln, Lincoln, UK \\ Email:mgoudas@pe.uth.gr
}

Received 8 October 2015; accepted 6 December 2015; published 9 December 2015

Copyright (C) 2015 by authors and Scientific Research Publishing Inc.

This work is licensed under the Creative Commons Attribution International License (CC BY).

http://creativecommons.org/licenses/by/4.0/

(c) (i) Open Access

\begin{abstract}
There is now strong evidence that exercise has an acute effect on the urge to smoke and the accompanying withdrawal symptoms. However, the perceptions by heavy smokers of exercise and its relationship to the urge to smoke have not been well documented. The aim of the present study is to understand the experiences of heavy smokers with regard to exercise and its effect on their urge to smoke. Five physically inactive, heavy smokers are asked to abstain from smoking the night before exercising on a cycle ergometer under two conditions (one at medium and one at vigorous intensity done a week apart). Semi-structured, in-depth interviews are conducted after the second exercise session. Thematic analysis reveals six themes describing the participants' experience of exercise, urge to smoke, exercise preferences, exercise and smoking relationship, exercise as an aid to quit smoking, and the effects of the experimental procedure. Overall, the participants' experiences support the existing literature, which has posited affective, biological, and cognitive mechanisms contributing to a delay in the urge to smoke after exercise. The main findings pertain to: (1) the "feel-good" effect after exercise as a relief from the "feel-bad" effect during exercise; (2) the decreased urge to smoke after exercise, stated by all participants regardless of reported positive and negative feelings; and (3) exercise as a "clearing the mind" mechanism rather than an attention-distracting mechanism.
\end{abstract}

\section{Keywords}

Physical Activity, Smoking, Perceptions, Intensity, Urge to Smoke 


\section{Introduction}

Physical activity and smoking are among those modifiable behaviors that have a positive impact on health. Today, there is abundant evidence for the positive effect of physical activity on health [1] [2]. However, only 15\% of Greeks take some forms of physical activity 5 times a week and only 3\% play sport at least once a week [3]. With regard to smoking, research has shown that it exacerbates the prevalence of various functional problems, leading to serious diseases and an increased mortality rate [4] [5]. Indeed, according to the HEART-funded Hellas Tobacco Survey in a nationwide household survey conducted in 2010, the prevalence of smoking in Greece was calculated at $41 \%$ ( $45 \%$ among men and $38 \%$ in women, $p=0.04)$. It is considered as one of the highest per capita consumption rates of tobacco products among European Union member states [6].

Although research examining physical activity and smoking behaviors started during the 1980s [7] [8], for some years the relationship between these behaviors was not clarified. Over the last decade, studies have supported an existing inverse relationship between the two variables [9] [10]. Despite the fact that studies of exercise-based treatments for substance use disorders have, in general, provided limited and inconsistent evidence, there are several theoretical and practical arguments in favor of such interventions [11]. Today, there are two groups of studies that have examined exercise interventions in relation to smoking: those examining the acute effects e.g. [12] and those examining the long-term effects e.g. [13]. Their respective results can enhance our understanding of how exercise can be used as treatment for smoking. However, there is little information from the acute-effect exercise studies about smokers' perception of exercise. Such information may improve the effectiveness of exercise-aided, smoking-cessation interventions.

In the last few years, three reviews on the acute effects of exercise on smoking-related measures were published: Haasova et al. [12], Roberts, Maddison, Simpson, Bullen, and Prapavessis [14], and Taylor, Ussher, and Faulkner [15]. Taylor et al. [15] reviewed 14 studies, 12 of which compared exercise bout with a passive condition; they reported a positive effect of exercise on cigarette craving, withdrawal symptoms, and smoking behavior. The other two studies compared exercise bout with two different intensities, revealing no differences in smoking-related outcomes. In all the studies reviewed, cigarette craving, withdrawal symptoms, and negative affect decreased during exercise and remained low for up to 50 minutes after it. Specifically, cravings and withdrawal symptoms were reduced after an exercise bout with intensity ranging from $60 \%-85 \%$ of the heart rate reserve (HRR) (lasting $30-40 \mathrm{~min}$ ) to $24 \%$ of the HRR (lasting $15 \mathrm{~min}$ ), and similarly with isometric exercise (for $5 \mathrm{~min}$ ). Taylor et al. [15] concluded that even relatively small doses of exercise should be recommended as an aid for managing cigarette craving and withdrawal symptoms. For future research, they suggested that the mechanisms involved, such as stress reduction and neurobiological mechanisms should be explored further because these could lead to the development of more effective and practical methods for managing withdrawal phenomena.

The review of Haasova et al. [12] used individual participants' data from 17 studies and compared participants engaging in physical activity with control group participants, using post-intervention measurements of strength of desire to smoke with baseline adjustments. Despite a high degree of between-study heterogeneity, their results showed physical activity groups achieving a greater reduction in cravings compared with the controls. The latest review by Roberts et al. [14] revealed similar findings: cigarette craving was reduced after exercise for a wide range of intensities, ranging from isometric exercise and yoga to vigorous activity of $80 \%-85 \%$ of the HRR. However, they reported that tobacco withdrawal symptoms and negative affect increased during vigorous exercise. They highlighted that, although we have evidence for the acute effect of exercise on reducing cigarette craving, it remains unclear which is the most effective exercise intensity to reduce the craving and what the underlying mechanisms associated with these effects are.

In an effort to identify potential mechanisms that could explain why exercise alleviates tobacco craving, Roberts et al. [14] clustered the most recent research findings into three hypothetical explanatory mechanisms: the affective, the biological, and the cognitive. According to the affective hypothetical mechanism, research evidence supports the claim that an increase in positive affect could result in a decreased desire to smoke. However, results from different studies disagree on the optimal level of intensity required to create an increase in positive effect, e.g., according to Bock, Marcus, King, Borrelli, and Roberts [16] and Harper [17] a bout of vigorous exercise reduced the negative affect and psychological withdrawal symptoms, respectively, in smokers undertaking an exercise-aided program for quitting smoking. Yet, in Everson, Daley, and Ussher's study [18], both moderate and vigorous intensity exercise conditions yielded similar effects on cravings and a notably adverse effect 
on mood. Everson et al. [18] suggested that perhaps moderate intensity exercise rather than vigorous exercise should be prescribed. They attributed the contradictory findings mainly to the participants' varying levels of motivation, and hypothesized that less tolerance to the vigorous exercise can be explained by the fact that these participants were abstaining from smoking for research purposes and so were not truly motivated to quit.

The biological hypothetical mechanism, which concerns the link between physiological exercise and smoking-related variables, remains uncharted territory. It is hypothesized that psychobiological changes (such as $\beta$-endorphins, opioids, and cortisol) can mediate the changes in withdrawal symptoms associated with exercise. Studies have supported changes in heart rate variability, caused by short or long abstinence and acute bouts of exercise, possibly having an immediate effect [19]-[21]. For example, a single session of exercise (e.g. a self-paced 15-min walk) can attenuate or reduce both post-exercise systolic and diastolic blood pressure responses to stress [22] [23].

The cognitive perspective, which claims that exercise may influence cognitive demands in such a way that it acts as a distraction from smoking-related thoughts, has not gained support recently due to no studies reporting an effect on distraction from cigarette craving [17] [24]-[26]. Roberts et al. [14] postulated that expectancy and credibility are two factors that need further examination as possible regulating factors in future research on the cognitive hypothesis. At the same time, the study [27] revealed varying cognitive activation of smokers towards smoking images after exercise when compared with control treatment. This finding suggests a neurocognitive process possibly being initiated after exercise, which mediates an effect on cigarette craving.

In conclusion, according to the research evidence it can be postulated that an acute bout of exercise reduces cigarette craving and other smoking-related variables, whereas part of the variation in the magnitude of the effects depends on the exercise intensity. But the question remains: How is this information linked to the knowledge that we need for promoting the adoption and maintenance of exercise among smokers who try to quit? To enrich our knowledge, we employed a qualitative methodological approach to explore answers to the following research question: How are acute bouts of exercise (of two different intensities) experienced by physically inactive, heavy smokers? We deemed that new information could possibly help researchers consider the potential pitfalls of acute exercise studies conducted in laboratory settings, because often laboratory effects do not translate well to the real world, when smokers try to quit using exercise as an aid. This approach can also provide a platform for rich participant-based information, from which new variables could emerge that act as potential moderators and mediators in the link between exercise and smoking behaviors.

\section{Methods}

The present study was part of a larger project aiming to examine the effects of exercise on physiological and psychological parameters related to smoking. The project consisted of a series of laboratory and field experiments which employed various exercise regimes.

\subsection{Participants}

The participants were five healthy Greek adults (four males and one female; mean age $36.00 \pm 3.39$ years), recruited through advertisements in the press offering a small monetary incentive to participate in the study. They obtained a doctor's permission to participate in the study. They were heavy smokers (mean number of cigarettes per day $24 \pm 5.48$; mean score on the Fagerstrom Nicotine Dependence Scale $6.80 \pm 1.64$ ) and physically inactive (as assessed by the International Physical Activity Questionnaire—short form-IPAQ, www.ipaq.ki.se), and were asked to abstain from smoking the night before the exercise. Their CO levels showed (PICO Smokerlyser, Bedfont, Rochester, UK) that they had indeed followed instructions and abstained from smoking ( $<15$ p.p.m. [parts per million], mean $=11.7 \pm 6.14$ p.p.m. for vigorous and $12.6 \pm 5.37$ p.p.m. for moderate exercise).

\subsection{Setting-Procedure-Data Collection}

Ethical approval for the current study was granted by the University of Thessaly Ethics Review Committee. Participants signed consent forms which informed them of their participation and withdrawal rights. They exercised on a cycle ergometer (Monark874E, Sweden) for 30 min under two different intensity conditions, with a 1-week interval between the two sessions. In one condition they were required to exercise at a medium intensity, maintaining their heart rate (HR) at an estimated 50\% - 60\% of their HRR. In the other they were required to exercise 
at vigorous intensity, maintaining their HR at an estimated $65 \%-75 \%$ of their HRR. The order of medium and vigorous exercise intensities was counterbalanced across the participants. Table 1 displays the physiological measures recorded during the two exercise conditions for each participant. In the vigorous-intensity condition the average HR and workload were higher and the average CO and carboxyhemoglobin levels were lower, although these values were the reverse of those seen in the medium-intensity condition.

Interviews were conducted immediately after completion of the second exercise session in a quiet setting. The duration of the interviews ranged from $45 \mathrm{~min}$ to $60 \mathrm{~min}$. The main interview questions were:

"How did you perceive the exercise experience you just completed?"

"What were your feelings/thoughts before and during the exercise session in comparison to what they are now-after the exercise?"

"Can you make a comparison between the two exercise sessions, last week and this week?"

"Being a smoker, do you think it affected your exercise experience? If yes, how?"

"Did the exercise sessions impact your urge to smoke immediately after the exercise? If yes, how?"

"If you chose to exercise in everyday life, what would you choose to do?"

"Would you in the future choose exercise as a supportive aid to quit if you decided to quit smoking?"

As the main perspective in qualitative research interviewing is personal meaning [28], the interviews conducted aimed to capture how the participants interpreted and understood the exercise experience through their own personal meaning. Follow-up probes were also used to promote dialogue and to obtain more detailed descriptions of the smokers' experiences during and after the exercise sessions, considering their perceptions, feelings, thoughts, interpretations, etc. Examples of probes include: “Can you tell me more about...?”, “Can you give me an example of...?” The interviews were conducted by the second author, they were audio recorded and then transcribed verbatim. The transcriptions yielded 72 pages of single-spaced text.

\subsection{Data Analysis}

Thematic analysis [29] was used to analyze the transcribed interviews. Analysis took place "at the end" [30]the coders-researchers analyzed the data after completion of data collection. Two of the authors read the transcriptions and identified higher-order themes, based on the research questions that had been developed initially. At the next step, lower-order themes within each higher-order theme were identified inductively. The data-driven lower-order themes were identified within the raw information and were grouped under each higher-order theme. The purpose was to search for patterns based on the information being studied [29]. During the categorization of the lower-order themes, the two authors discussed all themes exhaustively to reach a consensus. To enhance the reliability of themes and coding, a third author commented and the appropriate corrections were made until the three researchers had reached agreement.

Peer debriefing sessions were also conducted (with a researcher external to the study who was competent in qualitative methodology) to examine the methodological procedures and interpretations of the data coding [31]. To achieve trustworthiness [32], the following methods were used: (a) checking of members was carried out during and after the interviews; (b) the second researcher-interviewer kept notes of personal thoughts and nonverbal forms of communication during the exercise sessions and interviews, which comprised a reflexive

Table 1. Physiological measures for each participant and exercise intensity.

\begin{tabular}{|c|c|c|c|c|c|c|c|c|c|c|c|c|}
\hline \multirow[b]{3}{*}{ Participants } & \multicolumn{6}{|c|}{ Medium intensity } & \multicolumn{6}{|c|}{ Vigorous intensity } \\
\hline & \multicolumn{2}{|c|}{$\begin{array}{c}\text { HR } \\
\text { (beats/min) }\end{array}$} & \multicolumn{2}{|c|}{$\begin{array}{l}\text { WL } \\
\text { (W) }\end{array}$} & \multirow[t]{2}{*}{$\begin{array}{c}\text { CO } \\
\text { (p.p.m.) }\end{array}$} & \multirow[t]{2}{*}{$\begin{array}{c}\mathrm{COHb} \\
(\%)\end{array}$} & \multicolumn{2}{|c|}{ HR (beats/min) } & \multicolumn{2}{|c|}{ WL (W) } & \multirow[t]{2}{*}{$\begin{array}{c}\mathrm{CO} \\
\text { (p.p.m.) }\end{array}$} & \multirow[t]{2}{*}{$\begin{array}{c}\mathrm{COHb} \\
(\%)\end{array}$} \\
\hline & Mean & $\mathrm{SD}$ & Mean & SD & & & Mean & SD & Mean & SD & & \\
\hline Participant 1 & 133.33 & 6.86 & 88.10 & 11.36 & 7 & 1.6 & 148.17 & 15.05 & 96.53 & 12.36 & 5.5 & 1.5 \\
\hline Participant 2 & 128.83 & 6.85 & 107.05 & 9.12 & 15 & 3 & 142.67 & 5.43 & 101.33 & 11.38 & 20 & 3.4 \\
\hline Participant 3 & 127.50 & 7.69 & 83.73 & 18.92 & 7 & 1.8 & 151.83 & 6.97 & 109.00 & 22.26 & 6 & 1.6 \\
\hline Participant 4 & 127.83 & 6.55 & 75.53 & 8.24 & 19 & 3.7 & 138.83 & 14.91 & 106.95 & 17.40 & 15 & 3 \\
\hline Participant 5 & 133.00 & 6.00 & 84.63 & 12.85 & 15 & 3 & 142.33 & 16.51 & 102.50 & 16.76 & 12 & 2.6 \\
\hline
\end{tabular}

Note: CO: carbon monoxide; COHb: carboxyhemoglobin; HR: heart rate; p.p.m.: parts per million; SD: standard deviation; WL: workload. 
journal; (c) triangulation of the data [3] analysis was achieved by examining the participants' data on the physiological and psychological measures and the notes of the interviewer.

\section{Results and Discussion}

Analysis of the data produced the thematic structure shown in Table 2. Six higher-order themes were labeled: (1) experience of the exercise; (2) the urge to smoke; (3) exercise preferences; (4) the exercise and smoking rela-

\section{Table 2. Thematic structure of data analysis.}

\section{Experience of exercise}

During exercise

After exercise

\section{Affective}

Biological

Affective

Biological

Cognitive

Urge to smoke

During exercise

After exercise

$\begin{array}{lr}\text { Affective } & \text { Higher urge } \\ \text { Cognitive } & \text { Low urge } \\ & \text { Higher urge }\end{array}$

$\begin{array}{ll}\text { Affective } & \text { Low urge } \\ \text { Biological } & \text { Low urge }\end{array}$

\section{Exercise preference}

Preferred intensity

\begin{tabular}{lcc} 
& Vigor & Affective \\
& Moderate & Affective \\
Preferred type of exercise and & Cognitive \\
intensity in everyday life & Supervised & Indoors \\
& Gradual increase & \\
\multicolumn{1}{c}{ Exercise and smoking relationship } & \\
$\begin{array}{l}\text { Effects of exercise on } \\
\text { smoking behavior }\end{array}$ & Does not affect
\end{tabular}

Does affect

Effects of smoking on exercise behavior
Negative

Negative

Positive

Negative

Low urge

Not

Negative Hard, tired, unpleasant, pressure, wish to finish soon, negative thoughts

$$
\text { Positive Happy }
$$

Tired, exhausted, stressed, difficult, tension, weak

$$
\text { (immediately after) }
$$

Relaxed, satisfied, optimistic, better mood, positive, calm, more energy, more alive mentally and physically, more vivid, think better, active (after

$$
15 \text { mins) }
$$

Heavy and tired legs, hard even to walk, high heart rate, sweaty, painful

Is it because we forget or a relief from a false alert?

Stress, anxiety, need to relax from the tension (high intensity), pressure (high intensity)

$$
\text { Not thinking of smoking at all }
$$

$$
\text { As an escape from exercise pressure }
$$

Need to relax and calm down first, tired, exhausted, feelings of wellness, relaxed

Sweaty, out of breath, "open” lungs, breathing difficulties, need to rest first

No thoughts of smoking, clears your mind, not to waste the effort and satisfaction from accomplishment to finish the exercise

Better when I feel tense, difficult at first but then much better, more self-satisfaction, more enjoyable, gives more energy

$$
\text { More pleasant, easier, less tired }
$$

$$
\text { As beneficial as vigor }
$$

Walking, jogging, gardening

$$
\text { Gym }
$$

Gradual increase in both intensity and duration

$$
\begin{gathered}
\text { Unrelated behaviors, compatible behaviors } \\
\text { (if light physical activity or light smoker), } \\
\text { no effect on managing desire to smoke }
\end{gathered}
$$

Positive effect on general sense of self-control, increase in self-efficacy over behavioral control

Breathing problems, discomfort, low endurance, negative thoughts, difficult 


\section{Continued}

Exercise as an aid to quitting smoking

Would use under conditions

Moderate in intensity

Gradually increased intensity and duration

In combination with medicine

In combination with a strong will to quit

Reasons to use exercise as an aid to quitting smoking

Escape from sedentary routine
Feel healthier
Realize the harm of smoking
Social support/comparison
Empowerment to quit
Distraction from smoking
Feel-good effect

Effects of experimental procedure

On stress

During their first exercise experience, feelings of tension, stress

On urge to smoke

Increased urge to smoke due to unfamiliar environment

Momentum

Exercising as an experience to realize the negative effects of smoking on physical condition

tionship; (5) exercise as an aid to quitting smoking; and (6) the effects of the experimental procedure. A presentation of the themes follows, describing the meaning that participants attributed to the experience of not smoking from the previous night until after the morning of moderate- or vigorous-intensity exercise. Discussion of the findings has been incorporated into the results section to help the reader place the findings and their interpretations within the existing related literature.

\subsection{The Experience of Exercise}

Participants described their exercise experience using both positive and negative words. Comments related to their somatic condition were mostly negative about how they felt during the exercise or immediately after. Their responses reflected the increased fatigue or perceived exertion:

"During cycling I felt exhausted, but after I finished it feels OK, back to normal".

"In the beginning, I was feeling happy but as time was passing by I was very tired and wished to finish as soon as possible".

Similar experiences have been reported extensively by physically inactive smokers in previous research [24] [33] and are considered to be the main reason for limited compliance with the exercise programs [34]. Affective comments were also both positive and negative, with the negative ones referring to feelings during exercise as well us on completion, whereas positive ones referred to the period during which the participants' HR dropped. Similarly, in the relevant literature findings, exercise-related affective responses were positive [16] [35] or negative [18].

A comment made by a participant, who tried to explain why he thought he had negative feelings during exercise and positive ones after, could be a topic for further investigation:

"Is it because we forget? Maybe we think that it is OK when the pain (in the legs) is gone and then you say to yourself it is OK, it was nothing".

Thus, he explained that this happened possibly because he forgot the negative feelings he had during exercise with the relief of the positive feelings after the exercise, which compensated for the intense negative feelings during exercise. 


\subsection{Urge to Smoke}

Consistent with results from previous studies that suggest that exercise might reduce the urge to smoke and the accompanying withdrawal symptoms [25], most of our participants reported a decrease in the urge to smoke for a period ranging from $15 \mathrm{~min}$ to half a day. Participants' attributions for this delay in the urge to smoke related not only to affective and biological reasons but also to cognitive ones:

“... because you made a conscious effort to exercise and you felt satisfied with yourself that you did it".

It is interesting that both positive (e.g. wellness) and negative (e.g. exhaustion, tiredness) feelings were used as explanations for the decreased urge to smoke. When affect is changed during or after exercise (positively and/or negatively), it appears to decrease one's urge to smoke at that moment. Nevertheless, negative affect experienced after a vigorous exercise session can also have a detrimental influence on future exercise adoption and maintenance [35] [36]. According to a recent systematic review [37], a positive change in the basic affective response during moderate exercise was linked to future physical activity, but the post-exercise affect had a null relationship. They also found that affective responses during and after exercise correlated reliably with affective judgments about future physical activity. Other participants in our study provided answers that are more aligned with the biological approach, such as breathing difficulties and a sense of deep breathing, or "open" lungs as they described it. McClernon, Westman, and Rose [38] also detected this effect of (controlled) deep breathing on decreased withdrawal symptoms in dependent smokers.

A cognitive approach-related explanation was provided by some participants who elaborated on the absence of thoughts about smoking. The previous literature suggests that exercise acts as a mechanism that distracts attention; however, it was questioned whether this explanatory mechanism had been tested in an experiment [25]. In a research report, Daniel et al. [25] concluded that the effect of exercise in reducing both the desire to smoke and cigarette withdrawal symptoms is not caused by distraction. Based on contradictory findings, and due to the absence of adequate empirical evidence, further investigations should explore exercise as a "clearing the mind" procedure immediately after the exercise.

Some of our participants shared thoughts of smoking being present, but they tried to manage and control them. According to Ekkekakis and Acevedo [39], exercise acts as a thought suppressant, shifting one's attention from cognitive to somatic thoughts. The thought-suppression mechanism in smokers has generated quite controversial results in the literature. There are findings in support of the notion that thought suppression leads to a decreased desire to smoke [40], whereas other findings postulate that suppression of smoking thoughts results in increased subsequent smoking behavior [41]. Participants in the present study stated that they were trying to manage their smoking thoughts by rationalizing why they should not smoke after participating in an exercise session:

“... because you made a conscious effort to exercise and you felt satisfied with yourself that you did it".

It would be interesting, in future studies, to examine the effectiveness of suppression of smoking thoughts after an exercise session.

Although four participants reported a delay in the smoking urge, one of them indicated that he experienced an increase in his urge to smoke, especially during the vigorous exercise session:

“... the urge to smoke was higher before exercise as well as during exercise but lower after exercise".

Then he explained the increased urge as a reaction to the pressure that he felt during the exercise sessions. He specifically said:

"I would like to have a cigarette to relax from the tension of exercise".

Feelings of distress during vigorous exercise have been reported by smokers [18], and smoking is commonly explained by them as a stress management reaction [42].

\subsection{Exercise Preference}

With regard to the preferred intensity of the condition, most participants favored the medium intensity and explained their preference with both affective and cognitive statements, e.g.:

"I prefer the medium intensity, because as I said I am not used to exercise and it was an easier task for me than the intense".

The affective statements pertained to the differential feelings experienced during the two conditions, with greater emphasis on the more negative ones during vigorous exercise. Consistent with results from previous studies [18], moderate intensity was preferable because it was accompanied by more positive feelings and perceived benefits. The cognitive statement supporting the perceived positive benefits (or outcome expectancies) 
suggested that medium intensity was preferable. Outcome expectancies have been studied in the previous literature in relation to cravings or other tobacco withdrawal symptoms [17] [26], but not to the preferred exercise condition.

Preference for high-intensity exercise under specific conditions was reported by one participant who explained his choice through the affective perspective:

"... high intensity is preferable, difficult at first but then much better; the first 5 minutes makes you feel exhausted-tired causing breathing difficulties but after that point it was enjoyable. High-intensity exercise gives me more energy".

This participant was physically inactive, similar to the other participants, but during the interview he shared that he had been an athlete in the past. It is possible that the exercise-induced increase in his positive affect was triggered by his positive memory traces related to his past experiences as a competitive athlete. According to existing studies [37] [43], it is possible for an exerciser's memory to consciously or subconsciously influence the affect.

Statements about participants' preferences for physical activities in everyday life varied, depending on the type and duration of exercise; however, with regard to exercise intensity, most of the participants favored a gradual increase:

"I do not like to feel pushed (during exercise). I want to gradually increase the intensity".

Gradual increase in exercise intensity has been found to be beneficial for smokers who attended a program to quit smoking using exercise as an aid [16] [17]. When the participants attempted to explain their preferences over the type, duration, and intensity of exercise, their words reflected feelings of autonomy ("I would rather choose a mix of intensities according to my mood"), relatedness ("socializing while exercising is preferable"), and competence ("my physical condition is not good, so I easily get tired"). These quotes reflect a basic tenet of self-determination theory [44], which posits that one's intrinsic motivation for participation in a given activity is contingent on the satisfaction of one's basic needs of competence, autonomy, and relatedness.

\subsection{Exercise and Smoking Relationship}

Negative effects of smoking on exercise behavior were reported by the participants, e.g.:

"Cigarettes had an effect on my negative thoughts ... more negative thoughts than a non-smoker; it would be easier had I been a non-smoker".

These negative effects are consistent with what is found in the literature, for example [45]. However, two participants shared their physical activity and smoking behaviors being unrelated to each other, although the two behaviors coexist:

"I do not believe that exercise can make me stronger at managing my desire to smoke; it is just the good mood feeling at this moment".

When probed, they elaborated further that these behaviors can coexist when engaged in a lighter type of physical activity:

"I can smoke at the same time when gardening or biking, but it is not as enjoyable".

According to Boutelle, Murray, Jeffery, Hennrikus, and Lando [46], there may be a threshold level of exercise that must be reached for either physiological changes to occur in smokers' behavior or a negative effect on other behaviors to occur.

On the contrary, another participant stated that being physically active could be beneficial for his sense of self-efficacy and self-control, in general:

“... exercise had a positive effect on my perceived sense of control ... increased my sense of being effective over controlling my health behavior even if this is not the smoking behavior itself but maybe in general".

It should be noted here, and it is discussed again later, that this participant was the one who reported the momentum effect of the exercise experimental procedure on him outside the lab exercise behavior. It has been stated that changes in a harmful behavior (such as inactivity) may serve as a "gateway" for changing other behaviors (such as smoking), whereas increased self-efficacy, motivation, or self-confidence acts as a mediating mechanism [47]-[49].

\subsection{Exercise as an Aid to Quitting Smoking}

When asked if they would choose exercise as an aid to quitting smoking, most participants answered that they 
would use it under certain conditions:

"I would choose a moderate exercise due to my low fitness levels" or "Exercise might help me quit smoking but only if I had a strong will to quit".

The participants reported the following mechanisms with regard to how exercise might help them: the "feelgood" effect of exercise, distraction of attention, empowerment of changing one behavior for another (the "gateway" effect), routine changes to everyday life, recognition of benefits for a healthy lifestyle, realization of the harmful effect of smoking, and social support that they could get in group-based exercise programs. For example, one participant, when explaining how exercise might aid his effort to quit smoking, said:

“... because it would make me escape from the sedentary everyday routine and later, when I feel healthier and more active, then it might make me think that the smoking habit holds me back and I have to stop [smoking]".

Participants' statements about the link between their experiences of the acute effects of exercise and possible future attempts to quit smoking using exercise as a supportive method were related to the intensity of the proposed exercise program, their motivation to quit, and the additional support that could be received during the program (e.g. drug support). Existing empirical results suggest that the most effective programs for quitting smoking used a combined methods approach [50] [51], which appeared to be what our participants favored.

\subsection{Effects of the Experimental Procedure}

An unanticipated higher-order theme, relating to the experimental procedure itself, which appeared to have an effect on two participants, was identified in their answers without any probing or questioning. The theme was about the unfamiliar laboratory environment and experimental protocol, especially during their first visit, which pressured the participants:

"... during the first time, the lab, the experimental environment and procedure had an effect on the urge to smoke because I was unfamiliar and I felt a lot of pressure".

This might have had an effect on the exercise experience, which they shared with us. However, for another participant the experimental procedure had a different effect:

"I already started walking, four days now. I was never doing this before. The reason was: the first session was very tough on me when after 15 minutes on the bike I started feeling tired. And this made me think. And this thinking is working now a little and the only problem is smoking".

The first exercise session provided the momentum for him to continue, and soon after he realized that his fitness level was very low. This increased awareness motivated him to make an effort to increase his physical activity during the week between the two exercise sessions.

In general, the findings of the present study are in accordance with previous studies investigating the acute effects of exercise on abstinent smokers using a quantitative approach. Nevertheless, our findings also offer insights into and raise concerns about particular issues that should be taken into account when studying the mechanisms of acute exercise-induced effects on smokers' behaviors.

\section{Limitations}

Our study had several limitations: first, it is limited by its retrospective design for one of the two exercise sessions because the interviews were conducted after the second session. Participants were asked to recall their thoughts and feelings for the first of the two sessions in addition to the one they had just completed, which may have produced errors in inherent cognitive recall (especially errors of attribution) and time recall, and selective perception biases might have had an effect on their descriptions of what and when they felt or thought. However, by counterbalancing the order of medium and vigorous exercise intensities across participants and using probes for crucial answers, e.g. "How long after the end of the exercise session you ..." we partially controlled for these limitations and their effects on the data. A second limitation relates to the sample size, which was small, so the results could be limited in scope or applicability when addressing a wider population of smokers; therefore, it would be particularly useful in future studies to interview larger numbers of participants selected using a stratified sampling procedure, which will group participants according to key characteristics (e.g. stage of behavior change, motivation to quit, and exercise history), allowing comparisons at various levels.

\section{Conclusions}

In summary, our findings highlight the participants' interpretations of their experience of the acute effects of ex- 
ercise on their bodies, thoughts, and feelings. These interpretations and the meaning assigned to them are based on individuals' previous knowledge, experiences, emotions, beliefs, and attitudes about those two behaviors. Most of the interpretations have direct or indirect links to the existing literature in this research area. Participants' preferences for exercise intensity, type, and duration, in relation to the perceived effects of exercise on smoking urges, seem also to be related to individuals' past exercise experiences, general attitude, and beliefs. According to Stelter [52], personal meaning integrates the past, present, and future. It emerges, on the basis of earlier experiences, that the participant integrates into the current situation and, through this, integrates a possible or expected future into the current action. Overall, the variability of the participants' perspectives on the themes of exercise preference, exercise-smoking relationship, and exercise as an aid to quitting smoking seems to either directly or indirectly relate to their motivation to quit, and their stage of change for smoking and physical activity behavior. Moreover, there are some shared ideas that may be useful in generating possible future research ideas: a. the "feel-good" effect after exercise as a relief from the "feel-bad" effect during exercise; b. the decreased urge to smoke as an effect after exercise, stated by all participants regardless of reported positive or negative feelings; and c. exercise as more of a "clearing the mind" mechanism than one that distracts attention.

The main strength of the present study is the qualitative approach to a fairly well-researched topic, which until now involved quantitative approaches. By giving voice to participants, we are able to arrive at the personal meaning, which can lead to new research on the acute effects of exercise and the underlying mechanisms behind these effects.

\section{Acknowledgements}

Funding for this study was provided through the European Union (European Social Fund-ESF) and Greek national funds through the Operational Program "Education and Lifelong Learning" of the National Strategic Reference Framework (NSRF)—Research Funding Program: THALES. Investing in knowledge society through the European Social Fund.

We would like to thank Theodora Tzatzaki, Anastasia Tsiami, Eirini Manthou, and Kalliopi Georgakouli for running the exercise protocols and collecting the physiological data.

\section{References}

[1] Dishman, R.K., Washburn, R.A. and Heath, G.W. (2004) Physical Activity Epidemiology. Human Kinetics, Champaign.

[2] Khan, K.M., Thompson, A.M., Blair, S.N., Sallis, J.F., Powell, K.E., Bull, F.C. and Bauman, A.E. (2012) Sport and Exercise as Contributors to the Health of Nations. The Lancet, 380, 59-64. http://dx.doi.org/10.1016/S0140-6736(12)60865-4

[3] European Commission (2010) Special Eurobarometer: Sport and Physical Activity. Brussels.

[4] Doll, R., Peto, R., Boreham, J. and Sutherland, I. (2004) Mortality in Relation to Smoking: 50 Years' Observations on Male British Doctors. British Medical Journal, 328, 1519-1528. http://dx.doi.org/10.1136/bmj.38142.554479.AE

[5] Ezzati, M., Henley, S.J., Thun, M.J. and Lopez, A.D. (2005) Role of Smoking in Global and Regional Cardiovascular Mortality. Circulation, 112, 489-497. http://dx.doi.org/10.1161/CIRCULATIONAHA.104.521708

[6] Center for Global Tobacco Control (2011) The Greek Tobacco Epidemic. http://www.who.int/fctc/reporting/party_reports/greece_annex1_the_greek_tobacco_epidemic_2011.pdf

[7] Blair, S.N., Jacobs, D.R. and Powell, K.E. (1985) Relationships between Exercise or Physical Activity and Other Health Behaviors. Public Health Report, 100, 172-180.

[8] Conway, T.L. and Cronan, T.A. (1992) Smoking, Exercise, and Physical Fitness. Preventive Medicine, 21, $723-734$. http://dx.doi.org/10.1016/0091-7435(92)90079-W

[9] Paavola, M., Vartiainen, E. and Haukkala, A. (2004) Smoking, Alcohol Use, and Physical Activity: A 13-Year Longitudinal Study Ranging from Adolescence into Adulthood. Journal of Adolescent Health, 35, 238-244. http://dx.doi.org/10.1016/S1054-139X(04)00059-X

[10] Kaczynski, A.T., Manske, S.R., Mannell, R.C. and Grewal, K. (2008) Smoking and Physical Activity: A Systematic Review. American Journal of Health Behavior, 32, 93-110. http://dx.doi.org/10.5993/AJHB.32.1.9

[11] Linke, S.E. and Ussher, M. (2014). Exercise-Based Treatments for Substance Use Disorders: Evidence, Theory, and Practicality. The American Journal of Drug and Alcohol Abuse, 41, 7-15. http://dx.doi.org/10.3109/00952990.2014.976708 
[12] Haasova, M., Warren, F.C., Ussher, M., Janse Van Rensburg, K., Faulkner, G., Cropley, M., et al. (2013) The Acute Effects of Physical Activity on Cigarette Cravings: Systematic Review and Meta-Analysis with Individual Participant Data. Addiction, 108, 26-37. http://dx.doi.org/10.1111/j.1360-0443.2012.04034.X

[13] Ussher, M.H., Taylor, A. and Faulkner, G. (2012) Exercise Interventions for Smoking Cessation. Cochrane Database of Systematic Reviews, 1, Article ID: CD002295. http://dx.doi.org/10.1002/14651858.CD002295.pub4

[14] Roberts, V., Maddison, R., Simpson, C., Bullen, C. and Prapavessis, H. (2012) The Acute Effects of Exercise on Cigarette Cravings, Withdrawal Symptoms, Affect, and Smoking Behavior: Systematic Review and Meta-Analysis. Psychopharmacology, 222, 1-15. http://dx.doi.org/10.1007/s00213-012-2731-z

[15] Taylor, A.H., Ussher, M.H. and Faulkner, G. (2007) The Acute Effects of Exercise on Cigarette Cravings, Withdrawal Symptoms, Affect and Smoking Behavior: A Systematic Review. Addiction, 102, 534-543. http://dx.doi.org/10.1111/j.1360-0443.2006.01739.x

[16] Bock, B.C., Marcus, B.H., King, T.C., Borrelli, B. and Roberts, M.R. (1999) Exercise Effects on Withdrawal and Mood among Women Attempting Smoking Cessation. Addictive Behaviors, 24, 399-410. http://dx.doi.org/10.1016/S0306-4603(98)00088-4

[17] Harper, T.M. (2011) Mechanisms behind the Success of Exercise as an Adjunct Quit Smoking Aid. Doctoral Dissertation, Retrieved from Electronic Thesis and Dissertation Repository. http://ir.lib.uwo.ca/etd/198

[18] Everson, E.S., Daley, A.J. and Ussher, M. (2008) The Effects of Moderate and Vigorous Exercise on Desire to Smoke, Withdrawal Symptoms and Mood in Abstaining Young Adult Smokers. Mental Health and Physical Activity, 1, 26-31. http://dx.doi.org/10.1016/j.mhpa.2008.06.001

[19] Niedermaier, O.N., Smith, M.L., Beightol, L.A., Zukowskagrojec, Z., Goldstein, D.S. and Eckberg, D.L. (1993) Influence of Cigarette-Smoking on Human Autonomic Function. Circulation, 88, 562-571. http://dx.doi.org/10.1161/01.CIR.88.2.562

[20] Lucini, D., Bertocchi, F., Malliani, A. and Pagani, M. (1996) A Controlled Study of the Autonomic Changes Produced by Habitual Cigarette Smoking in Healthy Subjects. Cardiovascular Research, 31, 633-639. http://dx.doi.org/10.1016/0008-6363(96)00013-2

[21] Stein, P.K., Rottman, J.N. and Kleiger, R.S. (1996) Effect of 21 mg Transdermal Nicotine Patches and Smoking Cessation on Heart Rate Variability. American Journal of Cardiology, 77, 701-705. http://dx.doi.org/10.1016/S0002-9149(97)89203-X

[22] Hamer, M., Taylor, A.H. and Steptoe, A. (2006) The Effect of Acute Aerobic Exercise on Blood Pressure Reactivity to Psychological Stress: A Systematic Review and Meta-Analysis. Biological Psychology, 71, 183-190. http://dx.doi.org/10.1016/j.biopsycho.2005.04.004

[23] Taylor, A. and Katomeri, A. (2006) Effects of a Brisk Walk on Blood Pressure Responses to the Stroop, a Speech Task and a Smoking Cue among Temporarily Abstinent Smokers. Psychopharmacology, 184, 247-253. http://dx.doi.org/10.1007/s00213-005-0275-1

[24] Ussher, M., Nunziata, P., Cropley, M. and West, R. (2001) Effect of a Short Bout of Exercise on Tobacco Withdrawal Symptoms and Desire to Smoke. Psychopharmacology, 158, 66-72. http://dx.doi.org/10.1007/s002130100846

[25] Daniel, J.Z., Cropley, M. and Fife-Schaw, C. (2006) The Effect of Exercise in Reducing Desire to Smoke and Cigarette Withdrawal Symptoms Is Not Caused by Distraction. Addiction, 101, 1187-1192. http://dx.doi.org/10.1111/j.1360-0443.2006.01457.x

[26] Daniel, J.Z., Cropley, M. and Fife-Schaw, C. (2007) Acute Exercise Effects on Smoking Withdrawal Symptoms and Desire to Smoke Are Not Related to Expectation. Psychopharmacology, 195, 125-129. http://dx.doi.org/10.1007/s00213-007-0889-6

[27] Janse Van Rensburg, K., Taylor, A., Benattayallah, A. and Hodgson, T. (2012) The Effects of Exercise on Cigarette Cravings and Brain Activation in Response to Smoking-Related Images. Psychopharmacology, 221, 659-666. http://dx.doi.org/10.1007/s00213-011-2610-z

[28] Kvale, S. (1996) Interviews: An Introduction to Qualitative Research Interviewing. Sage, Thousand Oaks.

[29] Boyatzis, R.E. (1998) Transforming Qualitative Information: Thematic Analysis and Code Development. Sage, London.

[30] Rossman, B.G. and Rallis, S.F. (1998) Learning in the Field: An Introduction to Qualitative Research. Sage, London.

[31] Patton, M.Q. (1990) Qualitative Evaluation and Research Methods. 2nd Edition, Sage, Newbury Park.

[32] Lincoln, Y.S. and Guba, E. (1985) Naturalistic Enquiry. Sage, Beverly Hills.

[33] Daniel, J.Z., Cropley, M., Ussher, M. and West, R. (2004) Acute Effects of a Short Bout of Moderate versus Light Intensity Exercise versus Inactivity on Tobacco Withdrawal Symptoms in Sedentary Smokers. Psychopharmacology, 174, 320-326. http://dx.doi.org/10.1007/s00213-003-1762-x 
[34] Hughes, J.R., Crow, R.S., Jacobs, D.R., Mittelmark, M.B. and Leon, A.S. (1984) Physical Activity, Smoking, and Exercise-Induced Fatigue. Journal of Behavioral Medicine, 7, 217-230. http://dx.doi.org/10.1007/BF00845388

[35] Ekkekakis, P. and Petruzzello, S.J. (1999) Acute Aerobic Exercise and Affect: Current Status, Problems, and Prospects Regarding Dose-Response. Sports Medicine, 28, 337-374. http://dx.doi.org/10.2165/00007256-199928050-00005

[36] Dishman, R.K. (1990) Determinants of Participation in Physical Activity. In: Bouchard, C., Shephard, R.J., Stephens, T., Sutton, J.R. and McPherson, B.D., Eds., Exercise, Fitness, and Health: A Consensus of Current Knowledge, Human Kinetics, Champaign, 75-101.

[37] Rhodes, R.E. and Kates, A. (2015) Can the Affective Response to Exercise Predict Future Motives and Physical Activity Behavior? A Systematic Review of Published Evidence. Annals of Behavioral Medicine, 49, 715-731. http://dx.doi.org/10.1007/s12160-015-9704-5

[38] McClernon, F.J., Westman, E.C. and Rose, J.E. (2004) The Effects of Controlled Deep Breathing on Smoking Withdrawal Symptoms in Dependent Smokers. Addictive Behaviors, 29, 765-772. http://dx.doi.org/10.1016/j.addbeh.2004.02.005

[39] Ekkekakis, P. and Acevedo, E.O. (2006) Affective Responses to Acute Exercise: Toward a Psychobiological DoseResponse Model. In: Acevedo, E.O. and Ekkekakis, P., Eds., Psychobiology of Physical Activity, Human Kinetics, Champaign, 91-109.

[40] Erskine, J.A.K., Ussher, M., Cropley, M., Elgindi, A., Zaman, M. and Corlett, B. (2012) Effect of Thought Suppression on Desire to Smoke and Tobacco Withdrawal Symptoms. Psychopharmacology, 219, 205-211. http://dx.doi.org/10.1007/s00213-011-2391-4

[41] Erskine, J.A.K., Georgiou, G. and Kvavilashvili, L. (2010) I Suppress Therefore I Smoke: The Effects of Thought Suppression on Smoking Behavior. Psychological Science, 21, 1225-1230. http://dx.doi.org/10.1177/0956797610378687

[42] Kassel, J.D., Stroud, L.R. and Paronis, C.A. (2003) Smoking, Stress, and Negative Affect: Correlation, Causation, and Context across Stages of Smoking. Psychological Bulletin, 129, 270-304. http://dx.doi.org/10.1037/0033-2909.129.2.270

[43] Ekkekakis, P., Parfitt, G. and Petruzzello, S.J. (2011) The Pleasure and Displeasure People Feel when they Exercise at Different Intensities. Decennial Update and Progress towards a Tripartite Rationale for Exercise Intensity Prescription. Sports Medicine, 41, 641-671. http://dx.doi.org/10.2165/11590680-000000000-00000

[44] Ryan, R.M. and Deci, E.L. (2000) Self-Determination Theory and the Facilitation of Intrinsic Motivation, Social Development, and Well-Being. American Psychologist, 55, 68-78. http://dx.doi.org/10.1037/0003-066X.55.1.68

[45] Sandvik, L., Erikssen, G. and Thaulow, E. (1995) Long Term Effects of Smoking on Physical Fitness and Lung Function: A Longitudinal Study of 1393 Middle Aged Norwegian Men for Seven Years. British Medical Journal, 311, 715-718. http://dx.doi.org/10.1136/bmj.311.7007.715

[46] Boutelle, K.N., Murray, D.M., Jeffery, R.W., Hennrikus, D.J. and Lando, H.A. (2000) Associations between Exercise and Health Behaviors in a Community Sample of Working Adults. Preventive Medicine, 30, 217-224. http://dx.doi.org/10.1006/pmed.1999.0618

[47] Emmons, K.M., Marcus, B.H., Linnan, L., Rossi, J.S. and Abrams, D.B. (1994) Mechanisms in Multiple Risk Factor Interventions: Smoking, Physical Activity, and Dietary Fat Intake among Manufacturing Workers. Preventive Medicine, 23, 481-489. http://dx.doi.org/10.1006/pmed.1994.1066

[48] King, T.K., Marcus, B.H., Pinto, B.M., Emmons, K.M. and Abrams, D.B. (1996) Cognitive-Behavioral Mediators of Changing Multiple Behaviors: Smoking and a Sedentary Lifestyle. Preventive Medicine, 25, 684-691. http://dx.doi.org/10.1006/pmed.1996.0107

[49] Sherwood, N.E., Hennrikus, D.J., Jeffery, R.W., Lando, H.A. and Murray, D.M. (2000) Smokers with Multiple Behavioral Risk Factors: How Are They Different? Preventive Medicine, 31, 299-307. http://dx.doi.org/10.1006/pmed.2000.0710

[50] Ferguson, J., Bauld, L., Chesterman, J. and Judge, K. (2005) The English Smoking Treatment Services: One-Year Outcomes. Addiction, 100, 59-69. http://dx.doi.org/10.1111/j.1360-0443.2005.01028.x

[51] Fiore, M.C., Jaen, C.R., Baker, T.B., Bailey, W.C., Benowitz, N.L., Curry, S.J., et al. (2008) Treating Tobacco Use and Dependence: 2008 Update, Clinical Practice Guideline, US Department of Health and Human Services, Public Health Service, Rockville.

[52] Stelter, R. (2008) Exploring Body-Anchored and Experience-Based Learning in a Community of Practice. In: Schilhab, T., Juelskjær, M. and Moser, T., Eds., Learning Bodies, Danmarks Pædagogiske Universitetsforlag, Copenhagen, 111129. 